Highlights from the chapters include, in Chapter One ("Travel and Exploration"), an interesting comparison of two charts published by the Hydrographic Office of the Royal Navy showing the changes that occurred to the island of Krakatoa as a result of the catastrophic August 1883 volcanic eruption. Chapter Two brings us many excellent maps representing "Knowledge and Science." The standout example in this chapter come from the $1846 \mathrm{An}$ Historical Atlas in a Series of Maps of the World as known at different periods constructed upon an uniform scale and colored according to the political changes of the period by Edward Quin. Readers familiar with this atlas know that it is a series of maps, each showing a more extensive world, as, over time, it became known to Europeans. The known world on the maps is light and beautifully colored, while the "unknown" parts of the world are shrouded under a dark cloud called "terra incognita." The author of this map's description in Treasures from the Map Room rightfully tackles the absurd notion projected by this atlas that the world outside of the European context was "unknown" until discovered by Europeans. Chapter Three covers "Pride and Ownership," highlighting the idea that maps can show pride in ownership, either of a place itself or of a map depicting a place. The highlight of this chapter is the image of a small portion of a tapestry map showing part of Worcestershire. This tapestry is one of a set of four, each measuring about $15 \times 20$ feet! The tapestries were created to decorate a house - a rare idea in the late $16^{\text {th }}$ century.

In Chapter Four we find "Maps of War," which is a topic that cannot be ignored in a book such as this. The maps discussed show a variety of means for using maps in wartime. Of note in this chapter is the portion of a stunning map showing trenches along the front in World War One France. Chapter Five, "The City in Maps," shows the reader how cities have been mapped through the centuries. The real gem in this chapter is the panorama of London before the great fire, drawn by Dutch cartographer Claes Visscher. The details of buildings and in scenes of London life at the time are exquisite-seeing this one map in person should be on the bucket list of most map lovers. Chapter Six is all about "Maps for Fun" covering, for the most part, tourist maps. The standout in this chapter is the map of Yellowstone National Park in Wyoming. Its depiction of the park landscape, including the hot springs (viewed facing south from the Mammoth Hot Springs entrance), shows the park in its early years. Finally, in Chapter Seven we find maps of "Imaginary Lands" an interesting mix of maps of places that could have been, of places that were imagined to be, and of real places depicted as somehow symbolic.

The maps chosen to illustrate each of the chapter topics were thoughtfully chosen as representatives of the versatility and power that maps, and their meanings, can have. The reproductions are of high quality, and details are easily deciphered. When a map is too large to fit in its entirety on the page, either a well-chosen detail of the map is shown or the map is allowed to spill over onto the next page. Treasures from the Map Room successfully reaches its goal of showcasing the treasures of the map collections at the Bodleian Library at Oxford University with an interesting and thoughtful presentation. The reader gets a sense of each map and its historical importance, and a sense that the maps shown barely scratch the surface of the tremendous treasures to be found in the collections.

I would recommend Treasures from the Map Room for libraries who collect generally on cartographic history, or as a gift for lovers of maps and cartographic history.

\title{
GIS TUTORIAL 1 FOR ARCGIS PRO
}

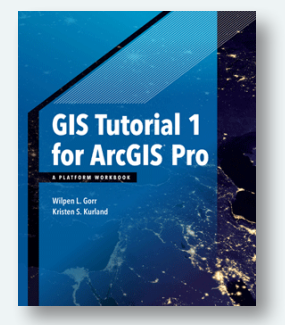

By Wilpen L. Gorr and Kristen S. Kurland

Esri Press, 2018

470 pages $\$ 99.99$, softcover.

ISBN: 978-1-58948-466-5

Review by: Tom Koch

\section{INTRODUCTION}

This latest instructional book from Esri Press is listed at \$99.99. Like its many predecessors, it serves three functions simultaneously. First, it seeks to teach the basic mechanics of an Esri software product, in this case ArcGIS Pro. Second, it seeks to sell Esri itself with the implicit message that GIS is Esri. Forget the many, and in some cases splendid, programs with first-rate instructional 
materials available elsewhere at either a lower price (for example, Maptitude), or for free (like QGIS). Third, as a "platform book," this volume serves as an introduction to, and thus promotion for, a range of other, individually costly (if potentially useful) Esri programs and apps.

Purchasers of GIS Tutorial 1 for ArcGIS Pro are offered a free, time-limited, introductory subscription to the software. The basic annual cost for a single user is USD $\$ 1,300$, a hefty price in a world where freebies like QGIS are multiplying. "Perpetual" licenses (which don't require annual renewal) are also available, with prices starting at USD $\$ 2,565$, as are academic and corporate subscriptions for multiple users. Costs of the latter types are available only on request from the Esri sales force.

\section{BASICS}

Why someone would choose ArcGIS Pro ("ArcPro") over another program (even ArcGIS Desktop) is something we're never told in this tutorial, although Esri User Conference demos of ArcPro present a potentially powerful program with extraordinary functionality for the handling of large datasets. It's not an easy program to learn: its menus are complex and sometimes confusing, and its command structure, to put it nicely, is complex. Anyone interested in using it likely needs, therefore, both a tutorial and time to learn a completely new set of commands.

That tutorial is what this 470 page tome sets out to provide. It is divided into four separate Parts, each containing between three and five chapters. The first 123 pages (Part 1) are about "Using, making and sharing maps" on the ArcPro platform. It includes instructions on downloading data for the course's examples, basic ArcPro techniques, and a general discussion of "map design" and "map concepts for GIS projects." The text assumes the reader knows nothing about maps, GIS software, or much of anything pertinent to the lessons it presents (I'll explain that last bit in a minute).

The 162 pages in Part 2 of the book, "Working with spatial data," describe the general construction and utility of Esri geodatabases (a way to hold and stabilize multiple datasets, feature classes, tables, rasters, and other, more complex entities), and spatial data in general, as well as ArcPro procedures for geoprocessing, digitizing, and geocoding. The third Part is "Applying advanced GIS technologies" with 115 pages on spatial analysis, raster GIS, and 3D GIS in
ArcPro, including data animations. Finally, Part 4 is titled "Managing operational systems with GIS." The tutorial projects in this part involve building a "Graffiti Mapping System" and a "Graffiti Mapping Removal System." The first analyzes patterns in infraction locations, and the second handles supervisory job tasking for clean-up crews. Since I'm not involved, at present, in either of these types of management activities, I didn't focus on these chapters.

Purchasers of GIS Tutorial 1 for ArcGIS Pro are first directed to a website where downloading of tutorial materials took 20 minutes on a relatively fast broadband connection. Installation run time for the downloaded .exe file was approximately fifteen minutes. The additional (and necessary) "assignments" download took a further 23 minutes and required about thirteen minutes of unpacking. ArcGIS Pro is significantly different from the company's principal product, ArcGIS Desktop, and those expecting an easy transition will be disappointed. The software itself has a somewhat daunting, complex, interface structure with multiple menus and dashboards that make for a steep learning curve, and also renders describing it the software challenging. All the controls are dynamic, so the reader has to get to the correct menu/sub-menu at the right time to find the option they need to select.

\section{OVERVIEW}

The book's type is generally quite small, especially what appears to be six-point type set on colored call-out boxes: a barely legible combination. I needed a magnifying glass to see the tiny menu icons embedded in the text. Finding anything in this book is difficult: there is no index, nor does the table of contents point the way if, say, a user wants to review specific protocols for changing a projection or adding a row to a database.

Like most Esri tutorials-for example, GIS Tutorial for ArcGIS 10: Spatial Analysis Workbook (Allen 2011)—the authors assume readers will have no previous knowledge of cartography or of mapping, either on- or offline. Thus, basics like coloration, resolution, scale, and spatial analytics are all offered as new material. It's hard, though, to imagine a neophyte going to this tutorial as a first experience in GIS. The question arises as to just what this book is supposed to be about: a tutorial for ArcGIS Pro, or a general introduction to GIS? A tutorial is normally limited to how to do something in a particular program. Trying to be all things to all readers only blurs the focus. Judged 
as an example of focused technical writing, it is clear that this volume was not written by experts in the trade.

That said, if one patiently follows the point-and-click instructions, one can, with practice, build a moderate competence in ArcPro. Some instructions are hard to follow, and in some places directions are unclear; but mostly, and with time, the tutorials can be more or less followed. I found it useful to create a set of short, "how-to" briefing notes as I worked through the book's examples. I will also admit that, while working through this tutorial, I modestly warmed to the program if not to this instructional volume. However, I still prefer my ArcGIS Desktop 10.4.

\section{TUTORIALS}

The tutorials evidence an arrogant and sometimes ignorant vacuity. We're told that "attributes play a major role in GIS," for example, and "Besides providing data needed to solve a problem or investigate spatial patterns, attributes allow you to search for useful information and mapped features" (19). The authors, both Carnegie Mellon policy and management academics - and authors of a previous Esri teaching book-should know better.

Attributes don't just "play a major role in GIS." They are the rows of data we collect and with which a stated problem is formulated; their organization and subsequent manipulation is the means by which a problem is addressed. Attributes don't let you search for "useful information." One can search for an individual datum, but as information theorists since Shannon (1948) have insisted, information is what we build in a specific context with the data we purposefully collect (Koch 2017b). Maps organize and project a set of selected "attributes" in an attempt to build information from data rows.

It is difficult to engage with, or care much about, the tutorial exercises because we are never told either the problem or the context. Take Tutorials 1.1-1.6, for example (33-46). We open a map that includes 14 symbolized layers that may have something to do with the accessibility of healthcare for citizens in the city of Pittsburgh and the surrounding Allegheny County, Pennsylvania. This could be an interesting, and even exciting, example of GIS analytics if we knew what the problem was, why these map layers were chosen, and why we were making this map.
Besides basic Pittsburgh-area geographies (city streets, county boundaries, rivers, and water features), layers include the locations of two different types of clinics (one private and one apparently publicly funded, sort of, maybe), poverty levels, and population density. Nowhere is there a problem statement: maybe this is about locating private clinics, maybe it is about healthcare; I don't know.

Whatever it is about, we also likely need to know the location of area hospitals whose emergency rooms serve as de facto clinics for millions of America's uninsured persons (EMTALA 1986). We might also wish to include ethnicity in the mix of variables: because the racial divide in the United States, and especially older cities like Pittsburgh, is often a critical determinant in the provision of health care and in the likelihood of a citizen having health insurance (for a review see Koch 2017b, Chapter Eight).

Worse, two of the layers describe buffers around the two different types of clinics. Both sets employ a one-mile radius, a size "commonly used," we are told, to determine accessibility to grocery stores in urban areas. "You can safely assume," the authors declare, "that what works for grocery store accessibility also works for health care facilities" (12).

Say what?

The term "grocery store" may describe anything from a large chain megastore (think Loblaws) to a small-time

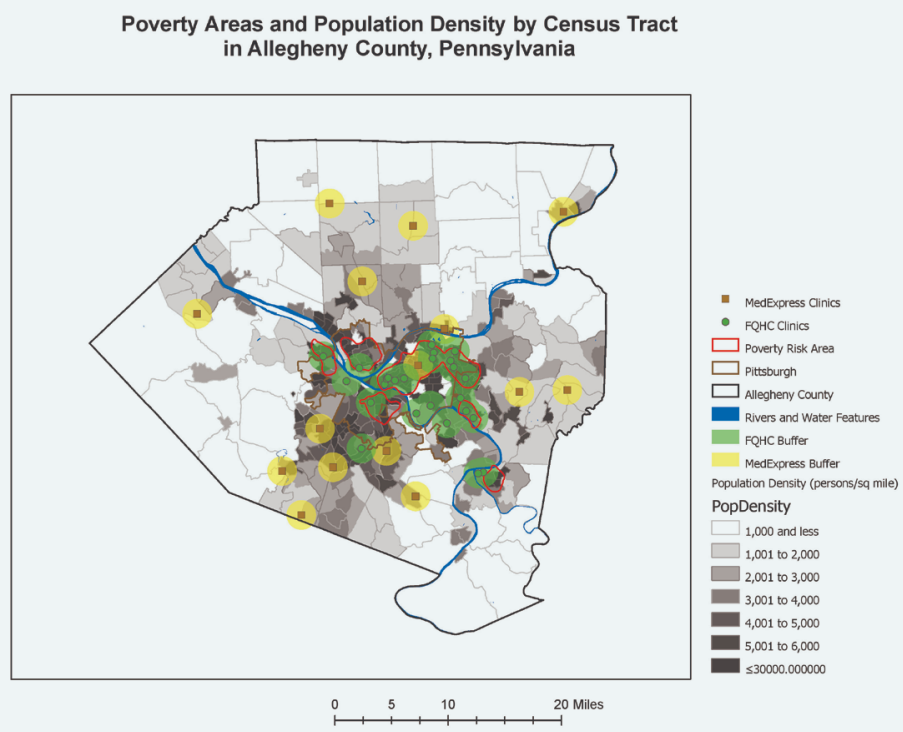

This is a map from a tutorial with various layers describing something-we're never told what-about health clinic accessibility in Pittsburgh, PA. 
local greengrocer. Across this range of providers, some will have fresh produce and some will not; some will overcharge for days-old bread and vegetables and others will have lower cost, fresher produce. Is the consumer walking, driving, or dependent on public transit? If they are driving, is there parking (at what cost?), and, if not, is there public transit available?

Location analysts spend their careers on this kind of problem. There is a huge literature on the location of both "food deserts," mostly in poor neighborhoods, and "food oases" where wealthier folk get the best quality produce at the best prices. Literally hundreds of papers have been written about these issues in the US public health literature (Story et al. 2008), health geography journals (Walker, Keane, and Burke 2010), and elsewhere. There are even articles dedicated to the mapping of food disparities (Phillips 2011). Nowhere in those literatures did I find anyone stating that a simple, one-mile buffer around any and all types of food outlets serves to answer any question.

The literature on health clinics and their availability is similarly complex, if not more so. All clinics are not the same. Some are run by physicians and some by nurses or nurse-practitioners. Some will have diagnostic equipment (EKGs, EEGs, ultrasound, for example) that others lack. Some will be able to handle complex infectious disease and trauma injuries. Others ... not so much.

Clinics are not simple and fungible. One size does not fit all. Do these two different clinic classes presented by the authors have different levels of competence and service? Can they be accessed by public transportation? Who knows? This is the type of management-speak arrogance that assumes mapmakers are mindless drudges (Wood 2002) who should just draw without question whatever data an employer or supervisor gives to them (Koch 2017a). It's also an example of oversimplified thinking that ignores a problem's real complexity to embrace simplistic formulations.

\section{DISCUSSION}

I've used one or another version of Esri software since 1996. Their new ArcGIS Pro program has potential, and some power users may find it their go-to GIS, especially if they prefer to work online. I am also an Esri Press author: they published both editions of my Cartographies of Disease and recently co-published my Ethics in Everyday Places. So I'm loathe to write a review that wholly dismisses one of their publications or any of their products. However, this book is an example of everything that is wrong with GIS when conceived as a mechanical, point-and-click exercise in which the mapmaker is assumed to be a mindless functionary. Its approach is shot through with the kind of magisterial, management-style assumptions that lead to poor analysis, faulty conclusions, and shoddy map making. Perhaps it could be used as an example to students of the kinds of clueless managerial oversight they can expect to encounter in their careers. Certainly any class of bright undergraduates could, given the opportunity, use the text's substantial failings to critique this kind of problem presentation. It would be a simple thing for the class to think up five problems to be considered based on the materials provided for Tutorials 1.1-1.6. I suspect too, that they'd also suggest other data sources to focus one or another problem more clearly.

To be fair, the authors Gorr and Kurland aren't technical writers. They're academics focused on public policy, management, and enterprise data analytics. That said, every chapter should start with a statement: "Here's the problem we want to address." It would be followed by a short description of the reasons specific datasets were collected and how they bear on the problem. After that, some remarks on the mechanics of making a simple map about something (coloration, resolution, scale, etc.) would make sense: why, for example, this projection rather than another? Later chapters in this volume deal with spatial analytics, and to deal with that we need to know the problem, what it is we are trying to argue or understand. Only then can the analysis become an intellectual rather than a robotic exercise. That, in turn, would add focus and clarity to the point-and-click instructions.

If the editorial focus of Esri Press were on how we use maps to consider problems and on what maps do, rather than on the software the company sells, we might get tutorials that were also terrific contributions to the literature-at-large. We might even have software that didn't become, with every iteration, less intuitive, more complex and ultimately more expensive. Alas, volume by teaching volume, Esri seems to be moving away from that mission. They didn't do it with this overblown book, and that is just too damn bad. 


\section{REFERENCES}

Allen, David W. 2011. GIS Tutorial for ArcGIS 10: Spatial Analysis Workbook. Redlands, CA: Esri Press.

Emergency Medical Treatment and Active Labor Act (EMTALA). 1986. 42 U.S.C. § 1395dd.

Koch, Tom. 2017a. Cartographies of Disease: Maps, Mapping, and Medicine, New enlarged edition. Redlands, CA: Esri Press.

. 2017b. Ethics in Everyday Places: Mapping Moral Stress, Distress, and Injury. Cambridge, MA: MIT Press and Esri Press.

Phillips, Anna L. 2011. "Making Better Maps of Food Deserts." American Scientist 99 (3): 209-210. doi: 10.1511/2011.90.209.
Shannon, Claude E. 1948. "A Mathematical Theory of Communication.” Bell System Technical Journal 27 (3): 379-423. doi: 10.1002/j.1538-7305.1948.tb01338.x.

Story, Mary, Karen M. Kaphingst, Ramona RobinsonO’Brien, Karen Glanz. 2008. "Creating Healthy Food and Eating Environments: Policy and Environmental Approaches." Annual Review of Public Health 29: 253-272. doi: 10.1146/annurev. publhealth.29.020907.090926.

Walker, Renee E., Christopher R. Keane, Jessica G. Burke. 2010. "Disparities and Access to Health Food in the United States: A Review of Food Deserts Literature." Health छ Place 16 (5): 876-884. doi: 10.1016/j.healthplace.2010.04.013.

Wood, Denis. 2002. "The Map as a Kind of Talk: Brian Harley and the Confabulation of the Inner and Outer Voice." Visual Communications, 1 (2) 139-161. doi: 10.1177/147035720200100201. 